\title{
Libration control of electrodynamic tethers using the extended time-delayed autosynchronization method
}

\author{
M. Iñarrea*and J. Peláez ${ }^{* *}$
}

\begin{abstract}
Any electrodynamic tether working in an inclined orbit is affected by a dynamic instability generated by the continuous pumping of energy from electromagnetic forces into the tether attitude motion. In order to overcome the difficulties associated with this instability, a new control scheme has been analyzed in this paper. The background strategy is as follows: we add appropriate forces to the system with the aim of converting an unstable periodic orbit of the governing equations into an asymptotically stable one. The idea is to take such a stabilized periodic orbit as the starting point for the operation of the electrodynamic tether. We use an extended delay feedback control scheme which has been used successfully in problems with one degree of freedom. In order to obtain results with broad validity, some simplifying assumptions have been introduced in the analysis. Thus, we assume a rigid tether with two end masses orbiting along a circular, inclined orbit. We also assume a constant tether current which does not depend on the attitude and orbital position of the tether. The Earth's magnetic field is modeled as a dipole aligned with the Earth's rotation axis.
\end{abstract}

\section{Introduction}

URING last years, new control techniques have been developed to be applied to nonlinear dynamical systems in order to transform chaotic or unstable behaviors into regular or periodic motions [13]. These new techniques have a goal: to achieve the control of chaos, that is, the possibility of bringing order into chaos. Some investigations have been undertaken using control schemes with and without feedback. However, the feedback control methods became a distinguished and important group among the plethora of different control techniques. Probably, the reason should be found in the advantage that they offer: they need comparatively small perturbations to get the control of the system, with respect to the non-feedback schemes (see [16]).

Pyragas in [18] proposed a feedback control scheme designed to synchronize the current state of a system and a time delayed version of itself. Taking this delayed time as the period of an unstable periodic orbit such a control scheme can be used to stabilize the orbit. This method of control is usually named time-delayed autosynchronization or TDAS. Two important advantages of this method are related with the feedback used: it does not requires rapid switching or sampling, nor does it require a reference signal corresponding to the desired orbit. This technique has been improved in $[17,15]$ using a more elaborated feedback: the extended time-delayed autosynchronization or ETDAS, where TDAS appears as a limiting case.

Frequently, dynamical systems exhibits unstable periodic orbits which usually appear embedded in chaotic attractors. Most of the research efforts were devoted to the control of chaotic behaviour

\footnotetext{
*Área de Física Aplicada, Universidad de la Rioja, 26006 Logroño, Spain. E-mail adclress: manuel.inarreaGunirioja.es

${ }^{* *}$ Grupo de Dinámica de Tethers (GDT). ETSI Aeronáuticos, Universidad Politécnica de Madrid, Madrid, Spain. E-mail address: j.pelaez@upm.es
} 
which can be found in many of these unstable orbits, a constant feature in low-dimensional dynamical systems. Such orbits can be controlled with small perturbation forces which decrease when time goes on because the system approaches the stabilized periodic orbit where they vanish. The instabilities considered are caused by various sources depending on the cases analyzed.

Control schemes using delayed feedback have been utilized in orbital and attitude dynamics of spacecrafts. One example of this kind of analysis can be found in [14] for a system with one degree of freedom. In that paper, a TDAS control law is used to stabilize the libration of a gravity-gradient satellite in an elliptical orbit; this kind of satellites exhibits a self-excited dynamics that is strongly chaotic in some regions of the parameter space.

There are some works trying to extend these techmiques to tethered spacecraft, especially when electrodynamic tethers are involved in the space system. Basically, an electrodynamic tether is made of a thin conductive wire. When flying in circular orbit the tether with zero current, has a stable equilibrium position on the local vertical. In the absence of damping or control, however, this gravity gradient stabilized equilibrium position disappears when the current begins to flow in the wire and the tether becomes unstable. Under some assumptions, the mathematical treatment simplifies and some characteristics of this instability can be drawn. This instability has been studied in previous analysis with different dynamic models (see $[11,12,6,9,5,8,10]$ ). They show that the instability source which drives any electrodynamic tether unstable is a non linear resonance mechanism that pumps energy continually into the system.

Eventually, the attitude motion of the tether relative to the orbital frame becomes unstable after several orbits. In those papers the tether current was assumed constant along the orbit and, in particular, independent of the actual tether position. This assumption, that we also adopt in this paper, permits to obtain results with broad validity which can be applied to any kind of electrodynamic tether regardless of the particular device used to collect electrons from the surrounding plasma.

For constant tether current, the analysis can be carried out as follows: instead of equilibrium positions, the governing equations exhibit periodic solutions with the period of the circular orbit followed by the system center of mass. In the absence of damping or control, these periodic orbits are unstable; the eigenvalues of the monodromy matrix of these periodic solutions provide a measure of the strength of such instability. The dynamic instability involved increases with the tether current and with the inclination. Consequently, it is quite natural to investigate the possibility of stabilizing such periodic orbits using the above mentioned techniques which have been specially designed to stabilize chaotic periodic orbits.

The analysis carried out by Peláez and Lorenzini in [3] is an attempt to applied these techniques to stabilize the attitude dynamic of electrodynamic tethers working at inclined orbits. They explore some control laws obtaining the families of periodic solutions which appear in the analysis and their stability properties by using a numeric algorithm which is described in Ref. [7]. An alternative procedure can be found in the work of Williams [2]. Therefore, the subject has interest by itself.

To lighten the analysis, we introduce some simplifying assumptions. Thus, the Earth's magnetic field is modeled as a dipole aligned with the Earth's rotation axis and we assume a rigid tether with two end masses orbiting along a circular and inclined orbit. Thus, the analysis does not include the response of the tether lateral dynamics which is also affected by the instability. However, the control of the librations is a necessary condition to the reliable operation of the tether; unfortunately, it is not sufficient. Since the coupling between librations and lateral modes is complex due to the electrodynamic forces (see Ref. [4] for a linear approximation), a further analysis would be required to asses the behaviour of the lateral modes.

In the paper [3] by Peláez and Lorenzini where they explore the possibility of use the TDAS method in this kind of problems, you can read: Unfortunately, the TDAS control scheme does not work appropriately in the problem of the electrodynamic tethers. Our calculations shows that this control law delays the instability but it does not stabilize the unstable periodic orbit for reasonable values of the control parameters.

A possibility to stabilize the tether with this kind of techniques is to use the extended time-delayed autosynchronization (ETDAS) method, which is an extension of the TDAS method. The ETDAS 
has been used with success in some cases where TDAS failed.

This paper is an attempt to extend the analysis of [3] by checking the extended time-delayed autosynchronization (ETDAS) method in some of the cases studied there. Our results are preliminary and they need to be confirmed by more detailed analysis. However, they are interesting and open the door to other control laws that permit to stabilize electrodynamic tethers which are not capable to be self-balanced.

\section{The uncontrolled tether and the basic periodic solutions}

In this paper, the tether is considered as a thin conductive rigid rod with length $L$ and mass $m_{t}$. A point mass $m_{B}$ is attached to the higher end of the tether. The orbiter $O$ is at the lower end. We assume the mass of the orbiter $m_{O}$ to be very large compared with the remaining masses of the system, that is, $m_{O} \gg m_{B}, m_{t}$ (this assumption could be easily removed as shown in [1]). The Earth's magnetic field is modeled as a perfect dipole aligned with the Earth's rotation axis. We focus the analysis on the system attitude dynamic and we neglect any decay in the orbit followed by the tether. As a consequence, we consider that the orbiter is tracing a circular orbit of radius $R \gg L$ and inclination $i$, with an angular velocity $\omega=\sqrt{\mu / R^{3}}$. See Fig. 1 (a). We assume that the tether current does not depend on the attitude or orbital position of the tether and therefore, the current keeps constant along the orbit.
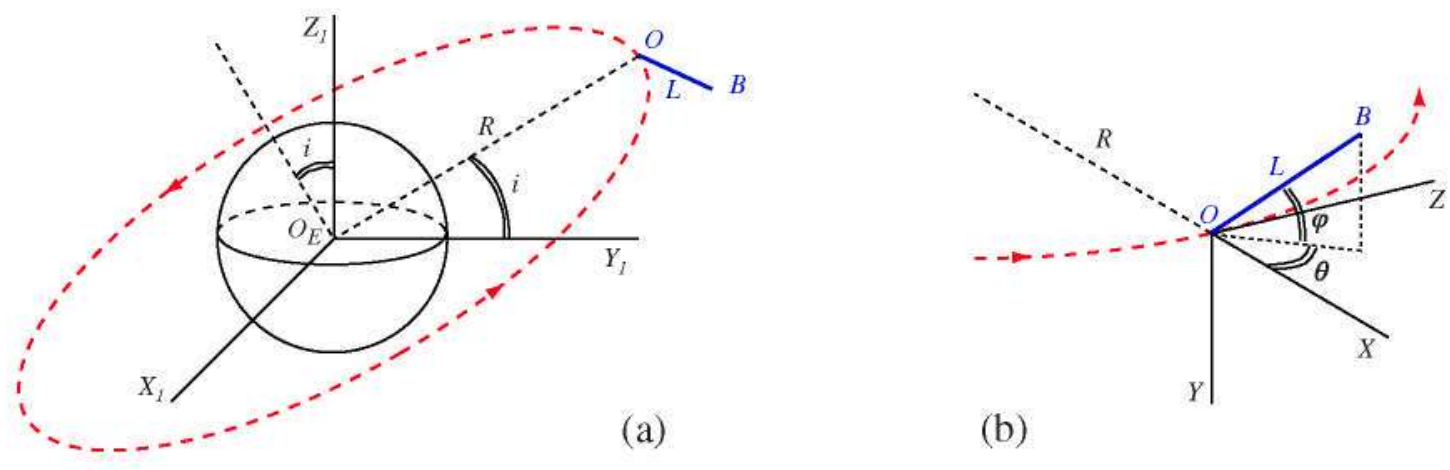

(b)

Figure 1: (a): Orbit traced by the tether. (b): Orbital reference frame and orientation of the tether.

We make use of two different right oriented orthonormal reference frames:

1. The inertial geocentric frame $\left\{O_{E}, X_{1}, Y_{1}, Z_{1}\right\}$ with the origin $O_{E}$ at the center of mass of the Earth, the $X_{1}$ axis pointing to the first Aries point, and the $Z_{1}$ axis aligned with the Earth's rotation axis.

2. The orbital frame $\{O, X, Y, Z\}$, with origin $O$ at the orbiter, the $X$ axis along the local vertical pointing to zenith, the $Z$ axis directed along the velocity vector of the orbiter, and the $Y$ axis normal to the orbital plane (Fig. 1(b)).

The orientation of the tether in the orbital frame is defined by two angles: the out-of-plane angle $\varphi$, formed by the tether and the orbital plane $(-\pi / 2 \leq \varphi \leq \pi / 2)$; and the in-plane angle $\theta$, formed by the $X$ axis and the projection of the tether on the orbital plane $(-\pi \leq \theta \leq \pi)$. See Fig. 1(b). These angles will be taken as generalized coordinates to study the attitude dynamics of the system.

Without control, the dynamics of the electrodynamic tether is governed by two kind of forces: the gravitational interaction and the electromagnetic one. The attitude dynamic relative to the orbital frame is governed by two torques: i) the one provided by the gravity gradient and the inertial Coriolis force and ii) the Lorentz torque due to the electrodynamic forces generated by the interaction 
between the tether current and the Earth's magnetic field. Taking into account these torques, the equations governing the librational motion of the tether take the following nondimensional form:

$$
\left\{\begin{array}{l}
\ddot{\theta}=2(1+\dot{\theta}) \dot{\varphi} \tan \varphi-\frac{3}{2} \sin (2 \theta)-\epsilon\left[\sin i \tan \varphi h_{1}(z, \theta)+\cos i\right] \\
\ddot{\varphi}=-\frac{1}{2} \sin (2 \varphi)\left[(1+\dot{\theta})^{2}+3 \cos ^{2} \theta\right]+\epsilon \sin i h_{2}(z, \theta) \\
\dot{\xi}=1
\end{array}\right.
$$

where

$$
h_{1}(z, \theta)=2 \sin z \cos \theta-\cos z \sin \theta \quad h_{2}(z, \theta)=2 \sin z \sin \theta+\cos z \cos \theta .
$$

Here, and along the paper, the dot means derivation with respect to the true anomaly $\nu$, measured from the lines of nodes $\nu=\nu_{0}+\omega t$. The variable $z$ has been introduced to make the system of differential equations autonomous. It is defined in one orbital period $\left[z_{0}, z_{0}+2 \pi\right]$, and it coincides with the true anomaly $\nu$ but for a constant. The tether current is on at the initial time $(t=0)$ and the equations must be integrated starting from the appropriate initial conditions

$$
\text { at } \nu=\nu_{0}(t=0) \quad \theta=\theta_{0}, \quad \varphi=\varphi_{0}, \quad \dot{\theta}=\dot{\theta}_{0}, \quad \dot{\varphi}=\dot{\varphi}_{0}
$$

These equations have been obtained using classical methods of analytical mechanics in the paper of Peláez and Lorenzini [3]. We will use the same notation because this analysis is an extension of the analysis carried out in that paper. The nondimensional parameter $\epsilon$ (see eq. [15] of paper [3]) describes the strength of the electrodynamic interaction; it vanishes for zero tether current and also for self-balanced electrodynamic tethers regardless of the value of the tether current (see [1]). In this way, the last terms in equations (1), including this parameter $\epsilon$, come from the Lorentz torque; the rest of the terms in equations (1) arise from the gravity gradient and inertial Coriolis forces. Therefore, the attitude dynamics of the tether depends only on two free parameters: the inclination angle $i$ of the orbit, and the electrodynamic parameter $\epsilon$.

For an inert tether, that is $\epsilon=0$, eqns. (1) exhibit steady solutions. In one of these singular points, the tether is aligned along the local vertical $(\theta=\varphi=0)$; this equilibrium position is stable. However, when $\epsilon \neq 0$, that is, when current is flowing through the tether, the steady solutions disappear. Assuming constant the value of $\epsilon$, instead of equilibrium positions eqns. (1) exhibit periodic solutions with the orbital period ( $2 \pi$ in the nondimensional time $\nu$ ).

The basic periodic solutions depend on the two free parameters $\epsilon$ and $i$ and they have been described in [3]; however, to increase the legibility of the paper we present here a brief summary. Figure 2 shows the form of these periodic solutions for different values of $\epsilon$ and $i$. In figure 2(a) the basic periodic solutions correspond to a small value of $\epsilon=0.5$ and several values of $i$ are depicted. For small values of $i$, the periodic solution is an oscillation in $\varphi$ with $\theta$ almost constant. For increasing values of $i$, the amplitudes of both angles grows noticeably. Figure 2(b) shows the basic periodic solutions for a greater value of $\epsilon=1.5$ and for the same values of the orbital inclination $i$. It is worth to note that when $\epsilon=0.5$, the amplitudes of the oscillations are quite small. In fact they are smaller than $15 \mathrm{deg}$. On the contrary, for bigger values of $\epsilon=1.5$ the amplitudes become significant, and they even can reach values close to $50 \mathrm{deg}$. From these pictures, it is clear that the amplitudes of both oscillations increase with $\epsilon$.

Apart from these basic periodic motions, it is important to note that the electrodynamic uncontrolled tether also exhibits more periodic solutions of eqns. (1). These other secondary periodic solutions have the same period $2 \pi$ and appear in pairs that are approximately symmetric with respect to the orbital plane. Moreover, there are more periodic solutions with period multiple of $2 \pi$. For more details about these secondary periodic libration motions, see Peláez and Lara [5].

The stability properties of the basic periodic solutions depend on the two free parameters $\epsilon$ and $i$. Nevertheless, when the system is not controlled all the basic periodic solutions are unstable for any value of $\epsilon$ and $i[11,12]$. The secondary periodic libration motions are also unstable, in fact, they are more unstable than the basic periodic solutions $[5,9]$. 

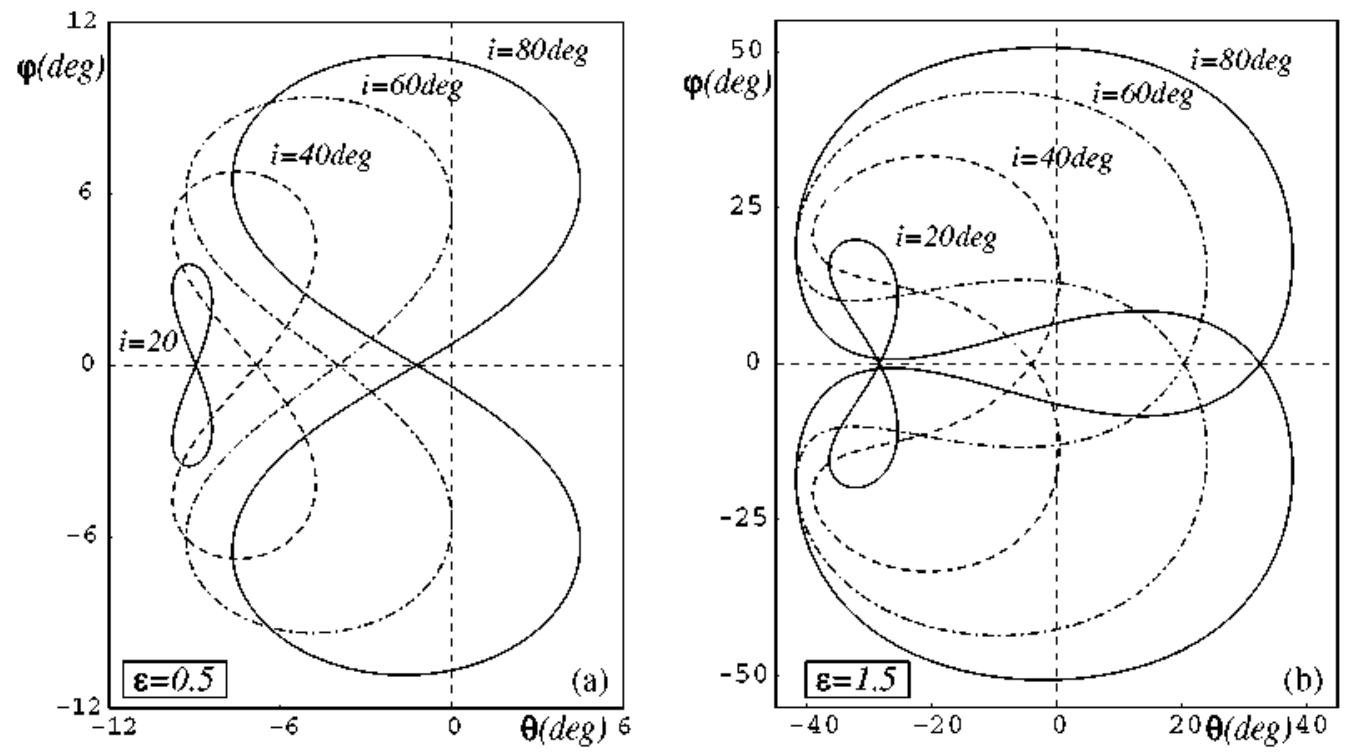

Figure 2: Basic periodic solutions for two values of the orbital inclination. (a) $\epsilon=0.5$. (b) $\epsilon=1.5$.
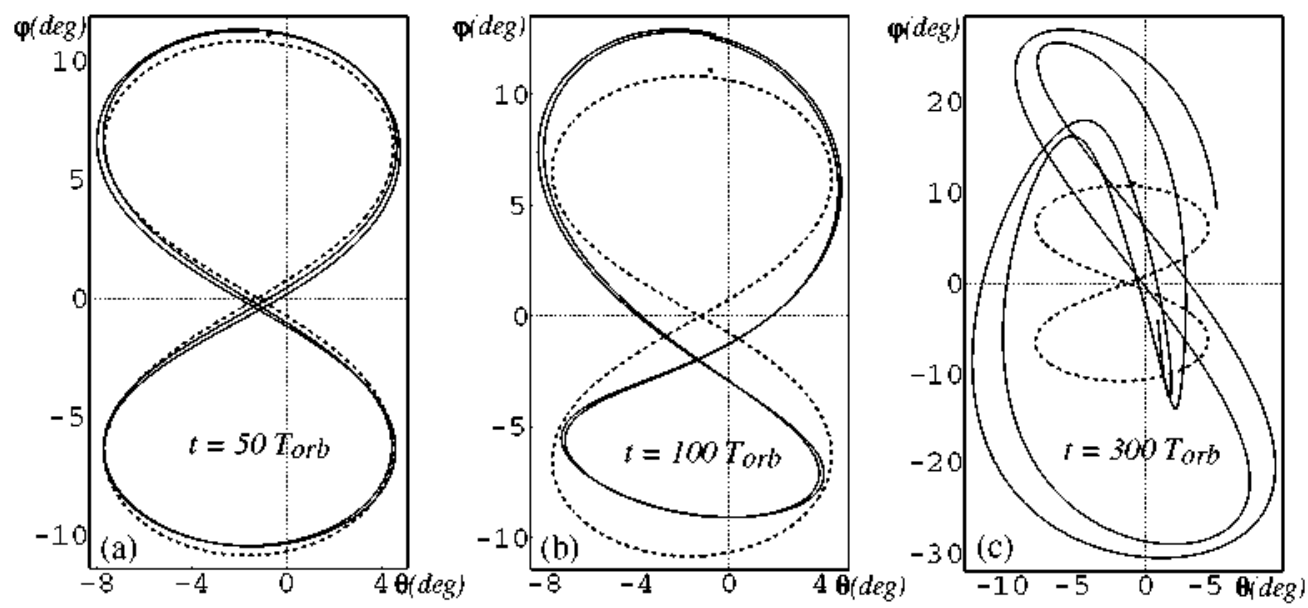

Figure 3: Unstable behavior of a libration motion with initial conditions very close to the basic periodic solution (dashed line) in the case $\epsilon=0.5$ and $i=80 \mathrm{deg}$ after different orbital periods. It is only represented the libration during the two last orbital periods.

Figures 3 and 4 show graphically two examples of the unstable character of the basic periodic motions of the uncontrolled tether. The dashed line represents the basic periodic solution, and the continuous line represents a libration motion starting from initial conditions very close to that periodic solution.

Figure 3 corresponds to the case $i=80 \mathrm{deg}$ and a small value of the electrodynamic parameter $\epsilon=0.5$. The plot represents the librational motion followed by the tether after 50, 100 and 300 orbital periods (only the last two periods are shown in the figure). From these graphs it is clear that although the motions start with initial conditions close to the periodic solution, after 300 orbital periods, the libration motion of the tether is very far away from the periodic trajectory. 
Figure 4 shows another example of the instability of the basic periodic solutions for a greater value of the electrodynamic parameter $\epsilon=1.5$ and $i=40 \mathrm{deg}$. In this case, the corresponding basic periodic motion is much more unstable. As it can be seen in figure 4 (b), after only 6.5 orbital periods, the libration motion of the tether is very far away from the periodic solution, in fact, the motion has suffered a transition from libration to rotation.

These two examples graphically reflect the fact that the instability of the basic periodic solutions strongly increases with the parameter $\epsilon$. An extensive analysis of the eigenvalues of the monodromy matrix of the periodic motions of the uncontrolled electrodynamic tether has been made by Peláez and Lara $[5,9]$. In this stability analysis, the authors study the dependence of the eigenvalues with the parameter $\epsilon$ and the inclination $i$. This study shows that the instability of the periodic solutions increase with the electrodynamic parameter $\epsilon$.
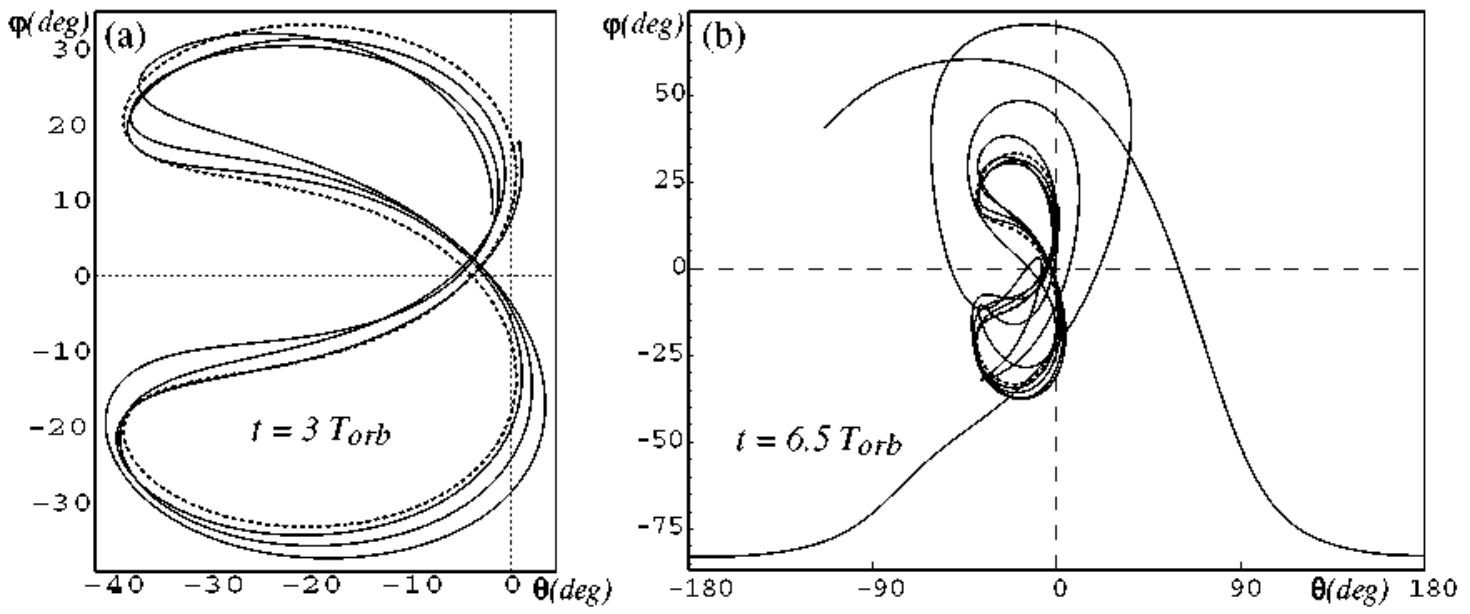

Figure 4: Unstable behavior of a libration motion with initial conditions very close to the basic periodic solution (dashed line) in the case $\epsilon=1.5$ and $i=40 \mathrm{deg}$ during different orbital periods.

\section{Libration control with the TDAS method}

The particular feedback control method that we use in this section is the so-called time-delay autosynchronization or TDAS [18]. This technique has two important advantages: it does not requires fast switching or sampling, nor does it needs a reference signal corresponding to the desired regular motion. It only requires the knowledge of the period of the desired periodic orbit.

The basic block diagram which describes the TDAS control techmique is shown in figure 5 . In the operation of this method, the control variable $y$ of the system is delayed at the output by some amount of time $\tau$, and then it is re-introduced into the system through the feedback control signal $F(t)=k\{y(t-\tau)-y(t)\}$. When consider periodic motions the delay time $\tau$ usually coincides with the period of the orbit. This control perturbation can be adjusted through the parameter $k$ in order to get the stabilization of the desired periodic orbit, that is, $k$ is free parameter of the problem. It is worth to point out that for any value of $k$, when the controlled system follows a periodic orbit of period $\tau$, the control signal $F(t)$ vanish, since in that case, $y(t-\tau)=y(t)$.

In the case of the electrodynamic tether, Peláez and Lorenzini [3], have applied the TDAS control method in order to convert those unstable periodic libration motions into stable periodic ones. It is important to note that a relevant feature of our physical model is a nonlinear resonance that pumps up energy continually into the system. In this way, we are dealing with a two degrees of freedom system that has a destabilizing mechanism which is different from the one usually found 


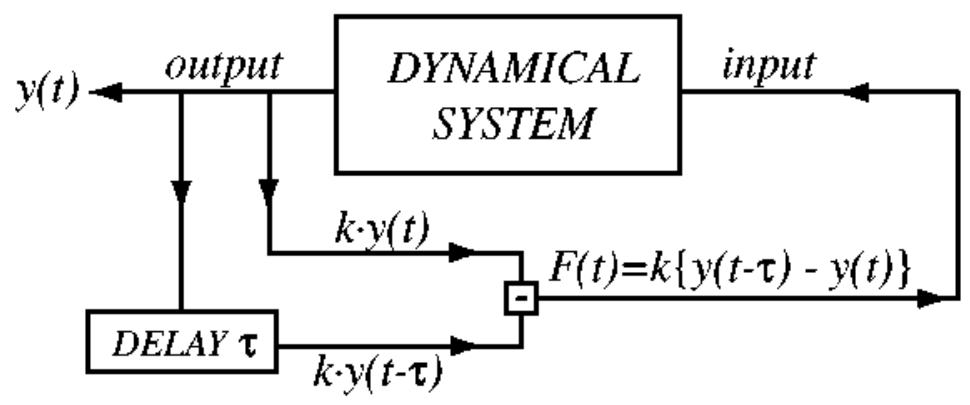

Figure 5: Block diagram of the TDAS control method.

in the literature of the control methods of nonlinear systems. In the study cited above, Peláez and Lorenzini assumed that the tether is acted upon additional forces, which introduce new terms in the governing equations in order to control effectively the tether dynamics. In this way, the TDAS control method they applied leads to the following governing equations for the controlled attitude motion:

$$
\left\{\begin{array}{l}
\ddot{\theta}=2(1+\dot{\theta}) \dot{\varphi} \tan \varphi-\frac{3}{2} \sin (2 \theta)-\varepsilon\left[\sin i \tan \varphi h_{1}(z, \theta)+\cos i\right]+F_{1}(\hat{z}) \\
\ddot{\varphi}=-\frac{1}{2} \sin (2 \varphi)\left[(1+\dot{\theta})^{2}+3 \cos ^{2} \theta\right]+\varepsilon \sin i h_{2}(z, \theta)+F_{2}(z) \\
\dot{z}=1
\end{array}\right.
$$

where the two control signals $F_{i}(z)$ are given by

$$
\begin{aligned}
& F_{1}(z)=k_{1}[\dot{\theta}(z)-\dot{\theta}(z-\tau)] \\
& F_{2}(z)=k_{2}[\dot{\varphi}(z)-\dot{\varphi}(z-\tau)] .
\end{aligned}
$$

In this case, since the system has two degrees of freedom, they added two control terms $F_{1}$ and $F_{2}$ to the equations of motion of the system. The variables of control they chose are the angular velocities $\dot{\theta}$ and $\dot{\varphi}$. The delay time $\tau$ must be precisely the period of the unstable periodic motions in the nondimensional time $\nu$, that is, $\tau=2 \pi$. In this way, there are two parameters $k_{1}$ and $k_{2}$ in the added control terms to get the stabilization of the basic periodic librational motions of the tether.

It is worth to point out that, when the controlled tether follows a $2 \pi$-periodic orbit, both control signals $F_{1}$ and $F_{2}$ vanish. Thus, any $2 \pi$-periodic motion of the uncontrolled system (1) is also a $2 \pi$-periodic libration of the controlled tether $(3)$. As a consequence, when the system moves in the neighborhoods of the periodic solution we should expect small values for the controlling forces $F_{1}$ and $F_{2}$ (in fact, they are torques acting on the system center of mass).

If this control method were to be successful, the basic periodic motion of the noncontrolled system (1) would become asymptotically stable when considered as a periodic libration of the controlled tether (3). In such a case, any motion of the controlled system starting in the attraction basin of that stabilized periodic libration, would approach it when times goes on. Therefore, after a while, the control terms becomes very small because they would tend to zero when $\nu \rightarrow \infty$. Thus, if from the very beginning the tether is operated close to the basic periodic solution, it can be controlled with small controlling forces. This is an attractive feature of this control method. In this way, the growth of the libration angles $\theta$ and $\varphi$ due to the instability of the uncontrolled system, could be removed by the control terms added to the governing equations.

Unfortunately, the numerous tests carried out by Peláez and Lorenzini in their study [3] showed that the TDAS control techmique fails to get the stabilization of the basic periodic motions of the tether. That is, in all those cases this control method has not been able to convert the unstable periodic motions of the uncontrolled tether into asymptotically stable ones. 
Figure 6 shows an example of this failure in the stabilization of one of those basic periodic orbits. The example corresponds to the case with parameters values $\epsilon=1.5$ and $i=40 \mathrm{deg}$. The figure shows the time evolution of the libration starting from initial conditions very close to the periodic orbit; several increasing multiples of the orbital period have been considered. In this example, the control parameters take the values $k_{1}=0.2$ and $k_{2}=1.2$. The controlled equations of motion (3) have been integrated for different combinations of the control parameters $k_{1}$ and $k_{2}$. Unfortunately, in all cases, the trajectory always moved away from the periodic motion after several orbital periods.

As Peláez and Lorenzini pointed out in their paper, the TDAS control scheme fails to stabilize the periodic motion because of the energy flow to the system coming from the electrodynamic interaction with the magnetic field of the Earth. Similar behavior has been found, for example, in a forced pendulum where the TDAS control method also fails [15].
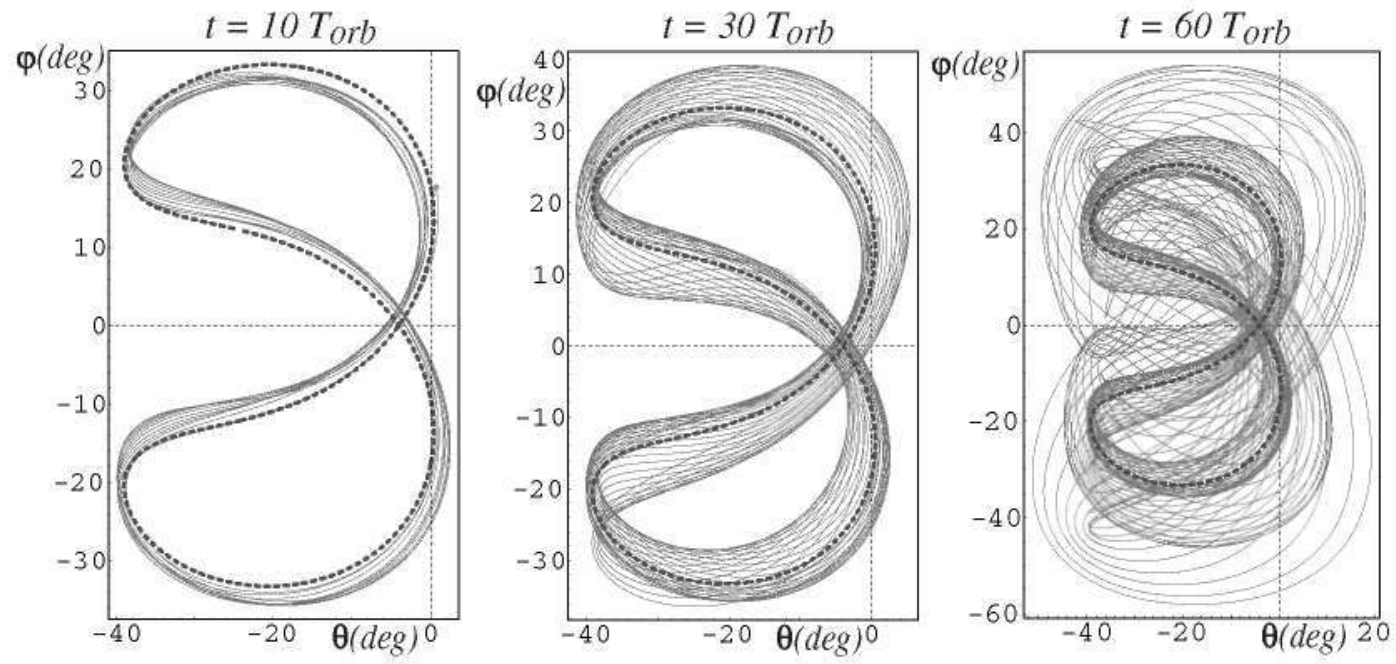

Figure 6: Failure of the TDAS control method for the case $\epsilon=1.5$ and $i=40$ deg. Libration motion after 10, 30 and 60 orbital periods. Control parameters $k_{1}=0.2$ and $k_{2}=1.2$. The dashed line represents the corresponding periodic motion.

\section{Libration control with the ETDAS method}

The failure of the TDAS method in the stabilization of the basic periodic orbits of the tether has moved us to apply a natural extension of this control technique, the so-called extended time-delay autosynchronization or ETDAS. This possibility was already mentioned by Peláez and Lorenzini at the end of their paper [3]. The ETDAS method was first proposed by Socolar et al. [17] to overcome the limitations of the TDAS technique in stabilizing periodic orbits. In this way, the ETDAS has been successfully applied in several systems were TDAS had previously failed $[15,17,19,16]$.

The basic block diagram of the ETDAS control method is shown in figure 7 . In the operation of this extended method, the control variable $y$ is progressively delayed at the output by multiples of some amount of time $\tau$. Then all these delayed control values $y(t-j \tau)$ are re-introduced into the system through the feedback control signal

$$
F(t)=k\left[y(t)-(1-R) \sum_{j=1}^{\infty} R^{j-1} y(t-j \tau)\right] .
$$

where $0 \leq R<1$ and $k$ are the two adjustable parameters of this control signal. 
When applied to periodic motion the delay time $\tau$ coincides with the period of the motion. In this way, the ETDAS method uses information of many previous states of the system in order to get the stabilization of the periodic orbit with period $\tau$. It is worth to emphasize that for any values of the control parameters $R$ and $k$, when the system follows a $\tau$-periodic orbit, the control signal $F(t)$ vanishes, because in that case, $y(t-j \tau)=y(t)$ for all $j$ (the identity

$$
\frac{1}{1-R}=\sum_{k=0}^{\infty} R^{k}
$$

has to be taken into account). Note also that, in the limit $R \rightarrow 0$, the ETDAS method coincides with the TDAS one.

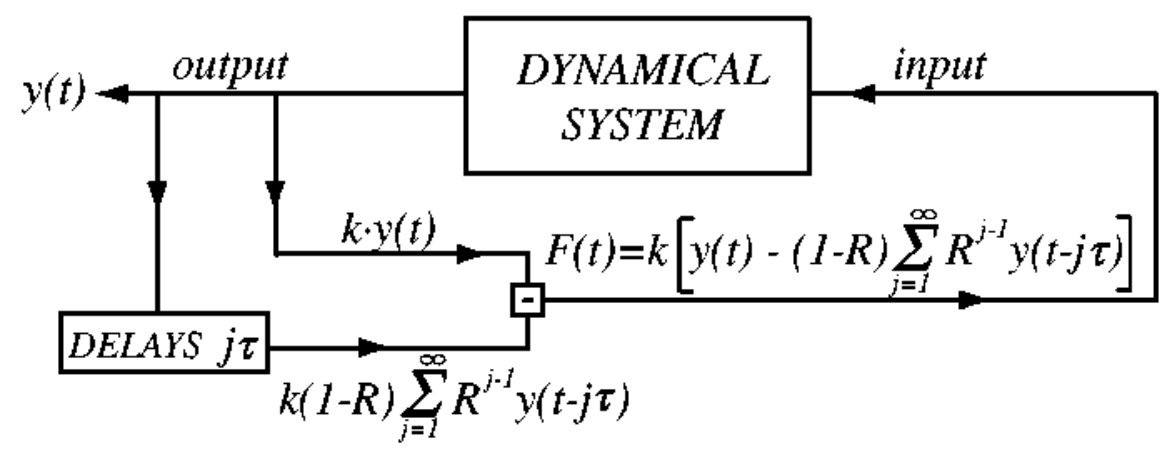

Figure 7: Block diagram of the ETDAS control method.

In order to stabilize the basic periodic librations of the electrodynamic tether, we have applied the ETDAS method in such a way that the governing equations of motion of the controlled tether take the same form as equations (3), where now the two control terms $F_{i}(s)$ are given by

$$
\begin{aligned}
& F_{1}(z)=k_{1}\left[\dot{\theta}(z)-\left(1-R_{1}\right) \sum_{j=1}^{\infty} R_{1}^{j-1} \dot{\theta}(z-j \tau)\right] \\
& F_{2}(z)=k_{2}\left[\dot{\varphi}(z)-\left(1-R_{2}\right) \sum_{j=1}^{\infty} R_{2}^{j-1} \dot{\varphi}(z-j \tau)\right] .
\end{aligned}
$$

Therefore, we have also chosen as control variables the angular velocities $\dot{\theta}$ and $\dot{\varphi}$. And we have four different adjustable control parameters, $k_{1}, k_{2}$, and $R_{1}, R_{2}$, with $0 \leq R_{i}<1$.

Figure 8 shows an example of the tests we have carried out integrating numerically the equations of motion controlled by the ETDAS method. This example corresponds to the same case shown in figure 6 with identical values of the parameters $\epsilon$ and $i$, and the same initial conditions very close to the corresponding periodic motion. In this case we have chosen as values of the control parameters $k_{1}=k_{2}=-0.2$ and $R_{1}=R_{2}=0.9$. The figure shows the evolution of the controlled librational motion during increasing multiples of the orbital period. For sake of clarity, in the graphs 8 (b) and (c) we have only represented the libration for the two last orbital periods. Figure shows the success of the ETDAS technique in the stabilization of the periodic orbits. In this case, as it can be seen in this figure, after 30 orbital periods the tether libration practically coincides with the basic periodic motion, in such a way that it is almost impossible to distinguish each other. This extended control method is able to change the dynamical character of the basic periodic motion, which has become asymptotically stable. We have found similar qualitative behavior for different values of $\epsilon$ and $i$. 

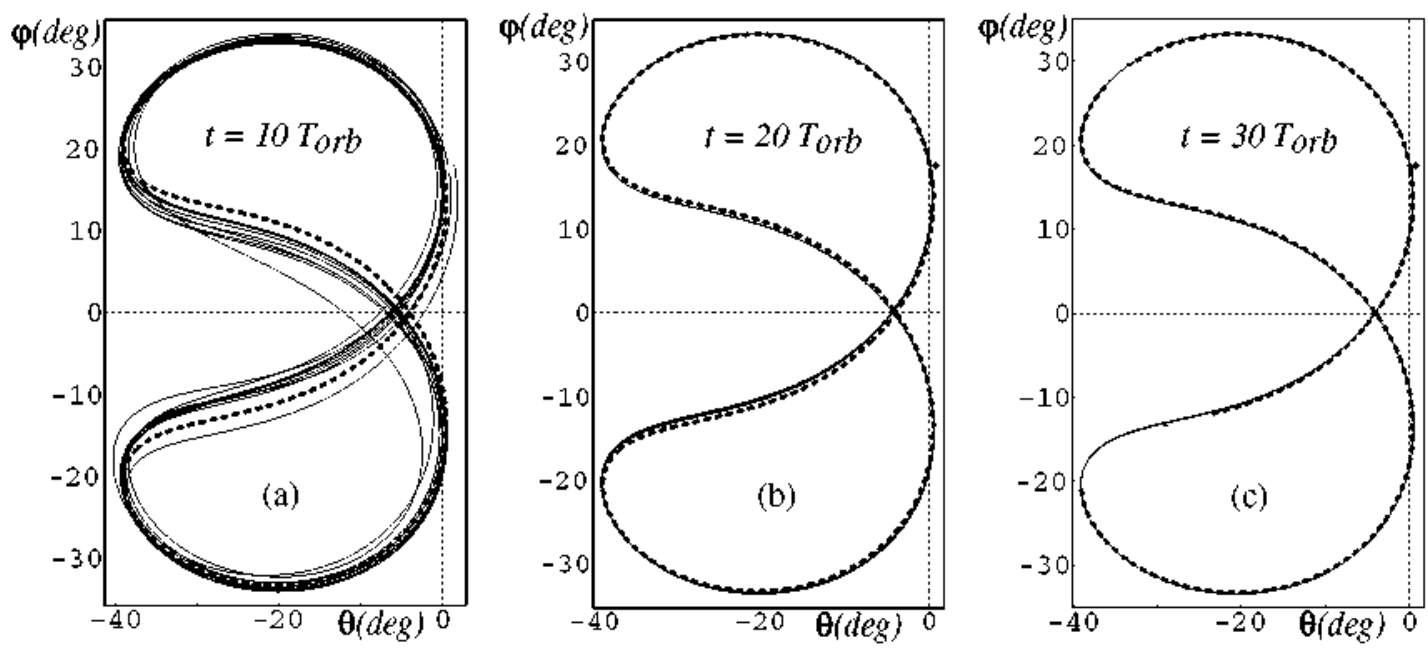

Figure 8: Success of the ETDAS control method for the case $\epsilon=1.5$ and $i=40 \mathrm{deg}$. Controlled libration motion after 10,20 and 30 orbital periods. Control parameters $k_{1}=k_{2}=-0.2$ and $R_{1}=R_{2}=0.9$. The dashed line stands for the corresponding periodic motion. In (b) and (c) it is only represented the libration during the two last orbital periods.

\section{Domains of control for the ETDAS method}

After having checked with several numerical tests that the ETDAS method is more powerful in stabilizing than the TDAS one, we have carried out the stability analysis of the basic periodic motions of the tether controlled by means of the ETDAS method. This stability analysis has been developed following the technique proposed by Blecih and Socolar [15].

Although this stability analysis method involves some cumbersome calculations, it has several important advantages. The method avoids the integration of the time-delay governing equations of motion of the controlled system. This integration would be a very delicate matter due to two non-trivial difficulties: first, the accuracy of the numerical integrator over long times and second, the choice of the initial conditions in the corresponding basin of attraction. The alternative method of stability analysis proposed by Bleich and Socolar only requires the integration of the equations of motion without the time-delay control terms $F_{i}$. Moreover, this integration must be carried out over only one period of the corresponding periodic motion. Basically, the method reduces to the calculation of the index around the origin of a curve in the complex plane.

By means of this stability analysis, we have calculated the domains of stability of the ETDAS control technique as functions of the control parameters, and for different values of the orbital inclination $i$ and the electrodynamic parameter $\epsilon$. For sake of simplicity, in this study we have take $k_{1}=k_{2}=k$ and $R_{1}=R_{2}=R$.

Figure 9 shows the domains of control in the parametric plane $(R, k)$ for several values of $\epsilon$ and $i$. Dark regions stand for the domains where the ETDAS method succeeds in stabilizing the periodic motion, whereas clear regions stand for the domains where the ETDAS fails. These domains of control have been calculated upon a two-dimensional grid of the control parameters $(R, k)$ with steps of 0.1 . We have carried out the stability analysis of the controlled periodic motion corresponding to each pair of the control parameters in order to know its dynamical character. This grid is transformed into a binary matrix depending on the stability of each controlled periodic motion for each pair of the control parameters. The resulting matrix is submitted as input to the commercial software TRANSFORM $[20]$ which produces the pictures in figure 9 by assigning the same color (dark or clear) to the same values of the binary matrix. 
Figure 9 also shows the evolution of the control domains for increasing values of $\epsilon$ and $i$. Some remarks must be pointed out about this evolution. On the one hand, when the orbital inclination $i$ increases, the domains of stable control decrease and, therefore the efficacy of the ETDAS method also decreases for any value of $\epsilon$. On the other hand, for a fixed inclination $i$, as the parameter $\epsilon$ increases, the domains of stable control also grow. This behavior seems to us quite paradoxical, as it means that, the more unstable the uncontrolled tether is (big values of $\epsilon$ ), the more effective the ETDAS method seems to be.

It is worth to note that this figure also includes the domains of control of the TDAS method, as the ETDAS one coincides with the TDAS in the limit $R \rightarrow 0$. Indeed, the TDAS domains of control are represented in the left limit $(R=0)$ of each parametric plane $(R, k)$ for $k_{1}=k_{2}=k$. In this way, we can also see in this figure 9 that the TDAS method is much less powerful than the ETDAS one. The TDAS fails in most cases shown in the figure, only in the case $\epsilon=1.5$ and $i=20$ deg the TDAS method succeeds for $k_{1}=k_{2}=k<0$.
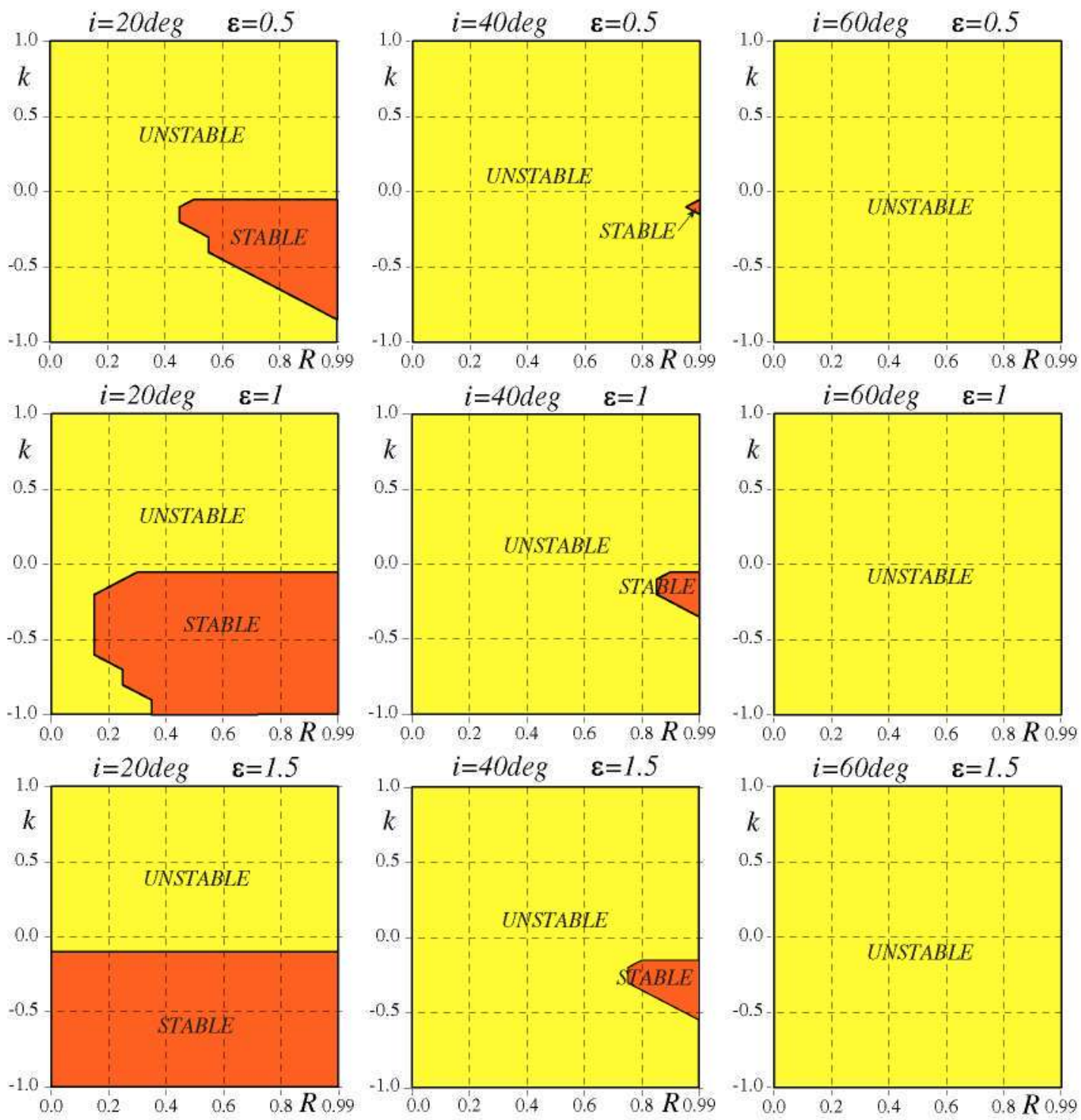

Figure 9: Evolution of the control domains of the ETDAS method in the plane $(R, k)$ of the control parameters as functions of $\epsilon$ and $i$. 
In order to check the validity of the calculated domains of control, we have carried out several numerical tests by integrating the controlled equations of motion for values of the control parameters $(R, k)$ belonging to each one of control domains. Figure 10 shows an example of these tests for the case $\epsilon=1$ and $i=25 \mathrm{deg}$. In figure 10 (b) it can be seen the corresponding domains of control in the parametric plane $(R, k)$. As representative examples of unstable and stable control we have chosen the values of the control parameters of point $A(0.1,-0.25)$ placed at the unstable domain, and by point $B(0.6,-0.25)$ situated at the stable one.

Stable region: figure 10 (c) shows the controlled tether libration after 90 orbital periods during only the last one for initial conditions very close to the corresponding periodic motion (dashed line), and for values of control parameters included in the stable control region (point $B$ ). In this case, we can observe the success of the ETDAS method as the controlled libration is almost indistinguishable from the basic periodic motion.

Unstable region: figure 10(a) shows the controlled libration after 600 orbital periods during only the last 100 ones, for the same initial conditions, and for values of control parameters inside the unstable control region (point $A$ ). In this other case, we may see the failure of the ETDAS technique in stabilizing the corresponding basic periodic motion, as the controlled libration moves away from it. Nevertheless, in spite of such a long time of integration, in this case we have not observed the characteristic transition from libration to rotation in the attitude motion of the uncontrolled tether. On the contrary, for even longer times of integration, we have found that the trajectory of the tether libration fills up completely the same region shown in figure 10 (a) without escaping from it.

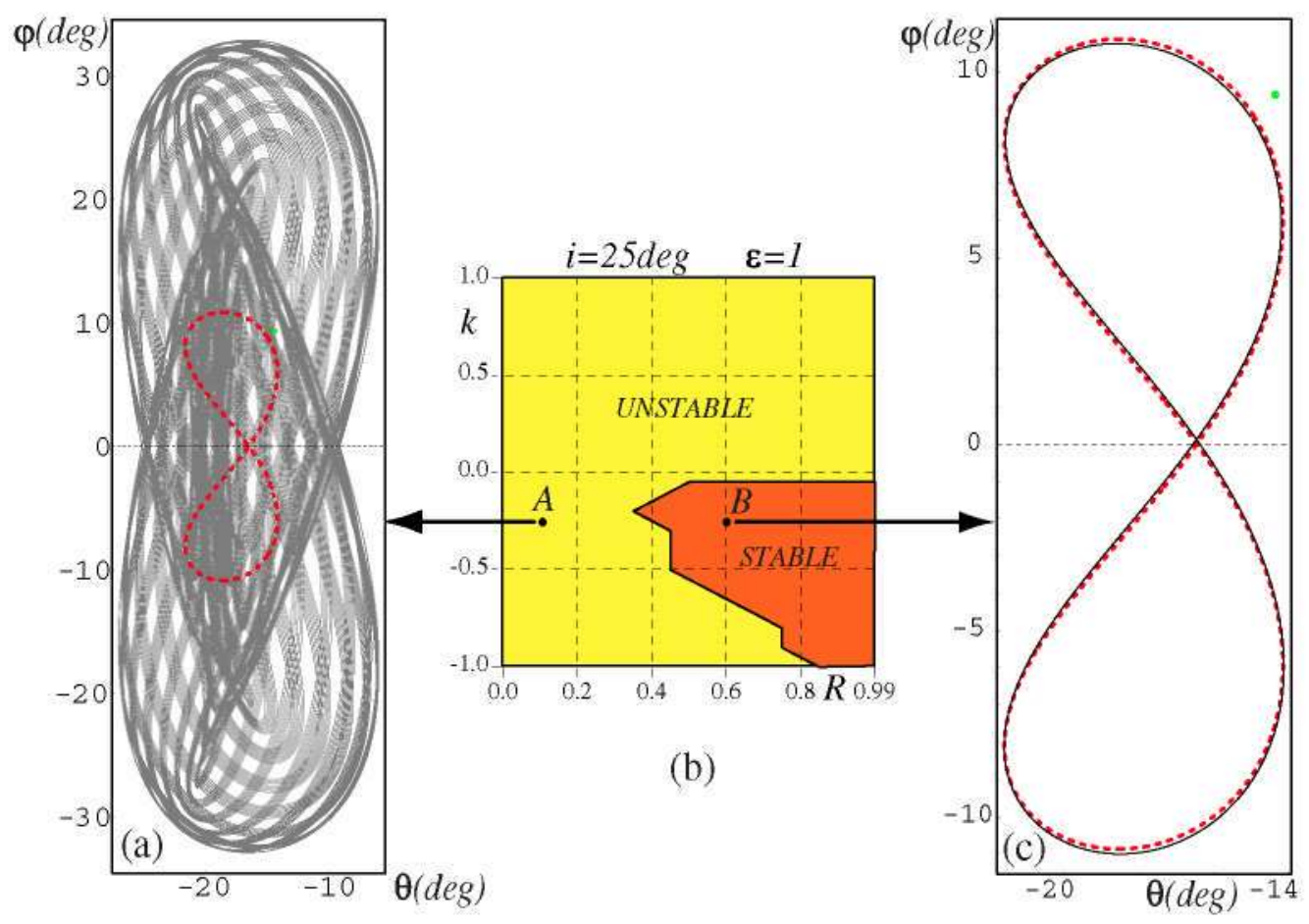

Figure 10: Two examples of the different efficacy of the ETDAS method in both domains of control for the case $\epsilon=1$ and $i=25 \mathrm{deg}$. (a) Unstable control for $R=0.1$ and $k=-0.25$. (b) Domains of control in the parametric plane $(R, k)$. (c) Stable control for $R=0.6$ and $k=-0.25$.

This curious behavior is not clear for us. We think that it is due to the existence of other secondary periodic librational motions in the dynamics of the uncontrolled tether (see [5]). As we have remarked in a previous section, these secondary periodic motions have the same period $2 \pi$ that the basic periodic ones, and they appear almost symmetrically with respect to the orbital plane 
$(\varphi=0)$. In this way, we think that although the ETDAS method fails in stabilizing the basic periodic motion, however it seems to be so powerful that succeeds in stabilizing one pair of those secondary periodic motions, in such a way that the trajectory of the controlled libration moves away from the basic periodic orbit, but it ends oscillating indefinitely between both symmetric secondary periodic motions filling up the region delimited by them. In any case, this behavior should be studied in detail in the future trying to clarify the reasons underneath it.

\section{Conclusions}

The application of the ETDAS method to control the libration motion of an electrodynamic tether in inclined circular orbit has been studied. The tether model we have considered is the classical dumbbell model. We have assumed that the tether currents is constant, the orbital decay is negligible, and the mass of the orbiter is very large with respect to the other masses of the system. The Earth's magnetic field is modeled as a dipole aligned with the rotation axis of the Earth. When the tether current is different form zero, the governing equations of the libration motion have periodic solutions that are all unstable for any values of orbital inclination $i$ and parameter $\epsilon$, which describes the strength of the electrodynamic forces.

In a previous paper [3], one of the authors studied the possibility of stabilizing the basic unstable periodic motions of the tether by means of a feedback control method: the time-delay autosynchronization TDAS. Unfortunately, that study showed that the TDAS control scheme is unable to stabilize those basic periodic librations. Following a final suggestion pointed out in that paper, we have investigated the application of a natural extension of the TDAS method: the extended time-delay autosynchronization or ETDAS. Both control techniques have two important advantages: they neither require rapid switching or sampling, nor need any reference signal corresponding to the desired periodic orbit, but only the period of it.

By means of numerical simulations of the libration motion of the controlled tether, we have found that the ETDAS method seems to be more powerful than the TDAS one. The ETDAS scheme succed in many cases where the TDAS fails in converting the basic unstable periodic motion of the uncontrolled system into an asymptotically stable orbit of the controlled tether.

In addition, we have also carried out the stability analysis of the basic periodic motions of the tether controlled by the ETDAS method. This stability analysis has been developed following the technique proposed by Bleich and Socolar [15]. In this analysis we have calculated the domains of stability of the ETDAS control scheme as functions of its control parameters $R$ and $k$, for several values of $i$ and $\epsilon$. The analysis confirms that the ETDAS method is much more efficient than the TDAS one in stabilizing the basic periodic motions. The study of the control domains has shown that paradoxically, the more unstable the uncontrolled tether is (big values of $\epsilon$ ), the more effective the ETDAS method seems to be.

We have also found that in the cases where the ETDAS scheme fails in stabilizing a basic unstable periodic solution, this control method seems to be able to stabilize a pair of secondary unstable periodic solutions which are symmetric with respect to the orbital plane and have the same period as the basic one. In these cases, the libration of the controlled tether moves away from the basic periodic motion and ends oscillating indefinitely between both symmetric secondary periodic motions. In this way, it is avoided the characteristic transition from libration to rotation that appears in the attitude motion of the uncontrolled tether.

Finally, the success of the ETDAS method stabilizing the electrodynamic tether in this particular case, opens the door to other different control schemes, also based in the ETDAS theory, but using the tether current as control parameter. 


\section{Acknowledgments}

The work of M. Iñarrea is included in the framework of the research project (MTM2005-08595) supported by the Spanish Ministry of Education and Science and by the Department of Education, Gobierno de Navarra, Spain (Project Resolución 18/2005). The work of J. Peláez was carried out in the framework of the research project entitled Dynamics of satellite orbit descent/raise using electrodynamic tethers (ESP2004-04376) supported by the Spanish Ministry of Education and Science.

\section{References}

[1] J. Peláez \& M. Sanjurjo, "Generator regime of self balanced electrodynamic bare tethers" Journal of Spacecraft and Rockets Vol. 43, (\# 6), November-December, 2006, pp. 1359-1369

[2] P. Williams, "Energy Rate Feedback for Libration Control of Electrodynamic Tethers", Journal of Guidance, Control and Dynamics, Vol. 29, No. 1, January-February 2006, pp. 221-223.

[3] J. Peláez and E.C. Lorenzini, "Libration control of electrodynamic tethers in inclined orbit". Journal of Guidance, Control and Dynamics, Vol. 28, 2005, 269-279.

[4] L. Somenzi, L. Iess \& J. Peláez, "Linear stability analysis of flexible electrodynamic tethers", Astrodynamics 2003, Advances in the Astronautical Sciences, Vol. 116, 2004, pp. 615-634.

[5] J. Peláez and M. Lara, "Periodic Solutions in Electrodynamic Tethers on Inclined Orbits", Journal of Guidance, Control, and Dynamics, Vol. 26, No. 3, 2003, pp. 395-406.

[6] J. Peláez, M. Ruiz, O. López-Rebollal, E. C. Lorenzini \& M. L. Cosmo, "A Two Bar Model for the Dynamics and Stability of Electrodynamic Tethers", Jounal of Guidance, Control and Dynamics, Vol. 25, No. 6, November-December 2002, pp. 1125-1135.

[7] M. Lara \& J. Peláez, "On the numerical continuation of periodic orbits: An intrinsic, 3Dimensional, Differential, Predictor-Corrector Algorithm", Astronomy and Astrophysics, Vol. 389, No. 2, July 2002, pp. 692-701.

[8] M. Dobrowolny, "Lateral Oscillations of an Electrodynamic Tether", The Journal of the Astronautical Sciences, Vol. 50, No. 2, April-June 2002, pp. 125-147.

[9] J. Peláez and M. Lara, "Periodic Solutions in Rigid Electrodynamic Tethers on Inclined Orbits", Advances in the Astronautical Sciences, Vol. 108, Pt. 2, 2001, pp. 1189-1208.

[10] J. Corsi \& L. Iess, "Stability and Control of Electrodynamic Tethers for De-orbiting Applications", Acta Astronautica, Vol. 48, No. 5-12, 2001, pp. 491-501.

[11] J. Peláez, E. C. Lorenzini, O. López-Rebollal and M. Ruiz, "A New Kind of Dynamic Instability in Electrodynamic Tethers", Advances in the Astronautical Sciences, Vol. 105, Pt. 2, 2000, pp. $1367-1386$.

[12] J. Peláez, E. C. Lorenzini, O. López-Rebollal and M. Ruiz, "A New Íind of Dynamic Instability in Electrodynamic Tethers", Journal of the Astronautical Sciences, Vol. 48, No. 4, 2000, pp. 449476 .

[13] S. Boccaletti, C. Grebobi, Y.-C. Lai, H. Mancini and D. Maza, "The Control of Chaos: Theory and Applications", Physics Reports, Vol. 329, 2000, pp. 103-197.

[14] Hironori A. Fujii, Wakano Ichiki, Shin-ichi Suda and Takeo R. Watanabe, "Chaos analysis on librational control of gravity-gradient satellite in elliptic orbit", Journal of Guidance, Control and Dynamics, Vol. 23, No. 1, January 2000, pp. 145-146. 
[15] M.E. Bleich and J.E.S. Socolar, "Stability of periodic orbits controlled by time-delay feedback", Physics Letters A, Vol. 210, 1996, pp. 87-94.

[16] K. Pyragas, "Control of Chaos via extended delay feedback", Physics Letters A, Vol. 206, 1995, pp. $323-330$.

[17] J.E.S. Socolar, D.W. Sukow and D.J. Gauthier, "Stabilizing unstable periodic orbits in fast dynamical systems", Physical Review E, Vol. 50, 1994, pp. 3245-3248.

[18] K. Pyragas, "Continuous control of chaos by self-controlling feedback", Physics Letters A, Vol. 170, No. 6, November 1992 , pp. 421-428.

[19] C. Batlle, E. Fossas and G. Olivar, "Extended time-delay autosynchronization of the buck converter", electronically available at http//arxiv.org/abs/chao-dyn/9609009.

[20] Fortner Research LLC, TRANSFORM, Sterling (1996). 